\title{
A Review on Vibration Damping in Sandwich Composite Structures
}

\author{
Zhuang $\mathrm{Li}^{\dagger}$ and Malcolm J. Crocker ${ }^{\dagger}$ \\ Department of Mechanical Engineering, Auburn University, Auburn, AL 36849, USA
}

(Received 29 April 2005; accepted 31 October 2005)

\begin{abstract}
In applications where the use of lightweight structures is important, the introduction of a viscoelastic core layer, which has high inherent damping between two face sheets, can produce a sandwich structure with high damping. Composite sandwich structures have several advantages, such as their high strength-to-weight ratio, excellent thermal insulation, and good performance as water and vapour barriers. So in recent years, such structures have become used increasingly in transportation vehicles and other applications. Care must be taken in their design to ensure that their sound isolation capabilities are adequate because coincidence generally occurs at a lower frequency for sandwich panels than for typical metal panels. Passive damping properties of composite sandwich panels are important because the damping properties affect their sound transmission loss, especially in the critical frequency range, and also their vibration response to excitation. Research on damping in sandwich composite structures is reviewed in this paper. This review includes analytical approaches, finite element models, statistical energy analysis, and damping measurement techniques. Other mechanical properties of composite sandwich structures, for example, stiffness and damage tolerance, affect each other and in turn are affected by damping. The overall effects of damping and other factors on structural response and sound radiation of composite sandwich structures are reviewed.
\end{abstract}

${ }^{\dagger}$ Member of the International Institute of Acoustics and Vibration (IIAV)

\section{INTRODUCTION}

A sandwich structure consists of three elements: the face sheets, the core, and the adhesive interface layers. The great advantage of sandwich structures is that optimal designs can be obtained for different applications by choosing different materials and geometric configurations of the face sheets and cores. By inserting a lightweight core between the two face sheets, the bending stiffness and strength are substantially increased compared to a single layer homogenous structure, without the addition of much weight. The viscoelastic core has a high inherent damping capacity. When the beam or plate undergoes flexural vibration, the damped core is constrained to shear. This shearing causes the flexural motion to be damped and the vibrational energy to be dissipated. Additionally, the normal-to-shear coupling between the core and face sheets reduces the sound transmission. So in recent years, such structures have become used increasingly in transportation vehicles and other applications. Rao has described the applications of viscoelastic damping in automotive and aircraft structures. ${ }^{1}$ Besides damping treatments used in structures, sandwich glass has been used in automotive side and rear windows to reduce noise. Composite materials are also used in pipes and ducts. ${ }^{110}$ Nakra has published a series of reviews on vibration control with viscoelastic materials. ${ }^{2-4}$ Trovik has summarised the major uses of constrained layer damping treatments up to $1980 .{ }^{5}$ A thorough review of work in fibre-reinforced composite material damping research has been given by Chandra et al. ${ }^{6}$ Some classical books and book chapters give more details on viscoelastic damping and sandwich structures. ${ }^{7-17}$

\section{ANALYTICAL THEORIES}

When a damping layer is attached to a vibrating structure, it dissipates energy by direct and shear strains. When a solid beam or plate is bending, the direct strain increases linearly with distance from the neutral axis. So, unconstrained damping layers, which dissipate energy mainly by direct strain, are attached to the remote surfaces. On the other hand, the shear stress is the largest at the neutral axis and zero on the free surfaces. Therefore, constrained layers dissipate energy by the action of shear stresses. It has been shown that shear damping in viscoelastic materials is higher than in typical structural materials. The constrained treatment has higher stiffness than the unconstrained damping treatment. For these reasons, sandwich composite structures are widely used.

\subsection{Damping in Sandwich Beams and Plates}

Since the late 1950 's, many papers have been published on the vibration of sandwich structures. The Ross-UngarKerwin model is one of the first theories which was developed for the damping in sandwich structures. ${ }^{18-21}$ In Kerwin's initial study, an analysis was presented for the bending wave propagation and the damping in a simply supported threelayer beam. ${ }^{18}$ One of the limitations of this analysis is that the bending stiffness of the top layer must be much smaller than that of the bottom layer. Ungar generalised the earlier study and derived an expression for the total loss factor of sandwich beams in terms of the shear and structural parameters. ${ }^{20}$ Based on such an expression, two important conclusions can be drawn. First, if the constraining layer is thinner than the viscoelastic damping layer, then the system damping has a

International Journal of Acoustics and Vibration, Vol. 10, No. 4, 2005

(pp 159-169) 